Commentary on the Paper of Walther L. et al.

Phosphatidylethanol (PEth) is superior to CDT and GGT as an alcohol marker and is a reliable estimate of alcohol consumption level

\author{
Wolfgang Weinmann ${ }^{1}$, Alexandra Schröck ${ }^{1}$, Friedrich Martin Wurst ${ }^{2,3}$ \\ ${ }^{1}$ Institute of Forensic Medicine, University of Bern, Bühlstr. 20, 3012 Bern, Switzerland \\ ${ }^{2}$ Paracelsus Medical University, Strubergasse 21, A-5020 Salzburg, Austria \\ ${ }^{3}$ Center for Interdisciplinary Addiction Research, University of Hamburg, Germany
}

Wolfgang.weinmann@irm.unibe.ch

Alexandra.Schroeck@irm.unibe.ch

Corresponding author:

Friedrich M. Wurst

Paracelsus Medical University,

Strubergasse 21

5020 Salzburg

Email: frieder.wurst@gmx.de

The paper of Walther et al. contributes to a timely and both from a scientific as well as clinical point of view relevant issue, namely the role of the ethanol metabolite phosphatidylethanol (PEth) in concert with questionnaires and biomarkers for the assessment of ethanol intake.

For a better understanding of strengths, potential limitations and future research needs, at first some aspects of the Alcohol Use Disorders Identification Test (AUDIT) and PEth are briefly recapitulated.

\title{
Alcohol Use Disorders Identification Test (AUDIT)
}

The 10-item AUDIT is a screening questionnaire developed by the World Health Organization (WHO) to identify harmful or hazardous alcohol consumption (Saunders et al., 1993).

The AUDIT-C, consisting of the first three questions of the AUDIT (quantity, frequency and binge drinking) was developed as an even briefer, easy to administer screening measure.

Both AUDIT and AUDIT-C are recommended by various guidelines such as of the National Institute for Health and Care Excellence, (NICE, UK; Jackson et al. 2010; NICE 2011), the Australian (Haber et al. 2009) and the German evidence- and consensus based guideline of the grade S3 (Mann, Batra 2015).

Neither the AUDIT nor the AUDIT-C are designed to provide a diagnosis of alcohol use disorder according to the International Classification of Diseases (ICD-10) or Diagnostic and Statistical Manual (DSM IV) criteria. Nevertheless, the probability of alcohol misuse or dependence is positively correlated to the AUDIT/AUDIT-C scores. Also, the first two questions allow an estimation of alcohol intake.

Of note, the AUDIT is a) retrospective and b) asks about typical consumption during the last year. This is of importance when correlating results of the AUDIT with data obtained from an alcohol diary and biomarkers, which might reflect different time spectra.

\section{Phosphatidylethanol (PEth)}

PEth is a phospholipid formed in the presence of alcohol via the action of the enzyme phospholipase D (PLD) (Alling et al., 1983). The precursors are naturally prevalent phosphatidylcholine homologues. The combined sum of the PEth homologues 16:0/18:1 and 16:0/18:2, which are the most prevalent ones in human blood (Gnann et al., 2014) correlates better with total PEth than each of them alone (Zheng et al., 2011).

PEth is formed after ingestion of alcohol and has a half-life time of approximately $4-10$ days (Varga, Hansson et al. 2000, Gnann, Weinmann et al. 2012).

During the last years, PEth has been employed in numerous settings such as assessment of driving ability, in forensic psychiatry, monitoring programs, and identification of alcohol intake in specific risk groups, e.g. for neonatal screening of prenatal alcohol exposure (for review, see Wurst et al. 2015). 
Recently, PEth has been recommended by the German Guideline on Alcohol Use disorders for screening of chronic alcohol intake with the highest level of evidence (level 1) in all settings for assessing drinking habits.

On this background, the paper discusses the use of alcohol biomarkers for the assessment of alcohol consumption levels in comparison to retrospective as well as diary-based alcohol self-reports.

For this study, AUDIT-C, drinking diary based self-reports (6 weeks), and alcohol state marker concentrations (PEth, CDT, GGT, AST and ALT) have been used to investigate correlations of alcohol biomarkers with the level of alcohol consumption.

The different biomarkers, questionnaires and self-reports represent different time frames. While the AUDIT and AUDIT-C ask for typical alcohol consumption during the last year, self-reports using a diary, reflect the daily alcohol intake of the last weeks. The alcohol biomarkers such as PEth, CDT and GGT have a detection window in the range of several weeks depending on the amount of consumed alcohol.

PEth - with a half-life time of 4 to 10 days - represents a period which is closer to the time of blood sampling. In cases of high daily doses, this will not make much of a difference, due to accumulation of PEth in blood. For assessing the sensitivity of the marker PEth, the patients have been grouped in three categories, those with intake of $0-49 \mathrm{~g}$ ethanol/day, $50-99 \mathrm{~g}$ ethanol/day and $\geq 100 \mathrm{~g}$ ethanol/day. Biomarkers have been determined at the start of study and in week 6 . Self-reported doses of ethanol at baseline (prior the start of the study) and in week 6 have been compared with AUDIT score, AUDIT-C score and the alcohol biomarkers. The reason for this classification however remains unclear and is obviously not based on frequently used classifications or recommendations, which would be of importance with regard to clinical meaningfulness and for potential comparisons.

Concerning PEth 16:0/18:1, the decision limits ("cut-offs") have been tested for their sensitivity for the reported alcohol consumption levels $(0-49 \mathrm{~g} /$ day, $50-99 \mathrm{~g} /$ day and $\geq 100 \mathrm{~g} /$ day). According to an agreement between Swedish laboratories the limits of decision for excessive alcohol consumption has been defined at $>0.3 \mu \mathrm{mol} / \mathrm{L}$ and for low or no alcohol consumption at $<0.05 \mu \mathrm{mol} / \mathrm{L}$ (Helander and Hansson, 2013). In addition, the limit of quantification (LOQ: $0.02 \mu \mathrm{mol} / \mathrm{L}$ ) of the used LC-MS/MS method for PEth detection was also tested as "cut-off" in this paper. For the group with low doses (0 $49 \mathrm{~g}$ ethanol per day) the sensitivity was $98 \%$ with a cut-off of $0.02 \mu \mathrm{mol} / \mathrm{L}$ PEth, and $93 \%$ for a cutoff of $0.05 \mu \mathrm{mol} / \mathrm{L}$ PEth. Since this low dose group also consisted of persons who had consumed only $22 \mathrm{~g}$ ethanol per day (roughly two standard drinks per day) as a minimum at baseline, and also no alcohol in one of the weeks before the last sample - it is not astonishing that a so called "false negative" rate - which on this background actually might be considered "correct negative" one, - was found at these cut-offs.

From our own drinking studies, we know, that at low drinking amounts (e.g. a single dose of ethanol, $35-66 \mathrm{~g}$, yielding a blood alcohol concentration (BAC) of $1 \%$ there is only minor accumulation of PEth, resulting in detection of low PEth values with a detection window of only 3 up to 12 days (Schröck 2014; manuscript in preparation).

For daily "high" - but not "extremely high" consumption, a group with daily doses of $50-99 \mathrm{~g}$ was defined by the authors. The sensitivity of the highest cut-off $(0.3 \mu \mathrm{mol} / \mathrm{L})$ for detection of high consumption seems to be very good with $84 \%$. Furthermore, all samples in this drinking category had PEth concentrations of above $0.05 \mu \mathrm{mol} / \mathrm{L}$ (100\% sensitivity). However, it is not quite clear, how the samples are distributed in this range. Furthermore, this definition is not in line with widely accepted ones, such as the NIAAA definition, were drinking more than the below mentioned single-day or weekly limits is considered "at-risk" or "heavy" drinking (NIAAA 2015): For men more than 4 drinks on any day or 14 per week and for women more than 3 drin ks on any day or 7 per week. Also, it does not match the WHO definition of high risk drinking with 61-100g for men and 41-60 $\mathrm{g}$ for women on a single day basis or $41+\mathrm{g}$ for women and $61+$ for chronic harm. (WHO, 2000). Of note, this issue is not limited to the work of Walther et al, but so many publications and thereby our knowledge on alcohol effects would benefit, if standardized and comparable definitions would be used. As indicated by the above mentioned NIAAA and WHO levels, gender specific aspects need to be taken into account. This is e.g. of importance with regard to the BAC reached for a certain alcohol intake given: According to Widmark equation, there is a large difference of BAC among individuals with different bodyweight, body water (gender: male/female, fat/thin, athletic). For example, the BAC reached by an $80 \mathrm{~kg}$ male compared to a $50 \mathrm{~kg}$ female when drinking the same amount of alcohol (in gram) can vary by factor 2 . 
Presuming similar elimination rates of ethanol, the area-under-the curve would differ enorumously, hence also the formed PEth concentrations.

In addition, the following points hold promise in being addressed in future research:

As PEth seems to be formed as long as alcohol is present in the blood, and higher BAC levels lead to a higher formation rate (shown by "in-vitro" experiments (Schröck 2015), the manner of drinking (speed, frequency, single drinking amounts) and the BAC reached per drinking event may play a crucial role in PEth production. This has not yet been investigated in detail.

Furthermore, it should be considered, that PEth is not only one molecule, but a family of phospholipids with different fatty acids in sn-1 and sn-2 position. Nutrition can influence the available lipids to be transformed to PEth homologues, and thus can play an important role for the occurrence of different fatty acid substituents.

No paper so far looked at degradation of PEth (in-vivo) and mechanism, how PEth is degraded and which products are formed. Apart from PLD activity, also PL A1 and A2, PL B and PL C can lead to different forms of "lyso"-PEth, which are currently not detected by LC-MS/MS methods. So, there is a variety of possibilities to explain inter-individual differences in PEth formation of certain homologues.

In conclusion, there is potentially a plethora of variables apart from daily amount of alcohol consumed, which influence PEth concentrations, such as gender (with influence on the area-under-the curve for blood alcohol concentration), nutrition and inter-individual differences in enzyme activities (for formation or degradation of PEth homologues), among others, which might be promising to be considered in future work.

\section{References}

Saunders JB, Aasland OG, Babor TF, de la Fuente JR, Grant M (1993) Development of the Alcohol Use Disorders Identification Test (AUDIT): WHO collaborative project on early detection of persons with harmful alcohol consumption. Addiction 88:791-804.

Haber P, Lintzeris N, Proude E, Lopatko O. Guidelines for the treatment of alcohol problems Australian Government Department of Health and Ageing, Sydney, 2009.

Jackson R, Johnson M, Campbell F, Messina J, Guillaume L, Meier P, Goyder E, Chilcott J, Payne N. Screening and brief interventions for prevention and early identification of alcohol use disorders in adults and young people. ScHARR, Scheffield, 2010.

National Institute for Health and Clinical Excellence (NICE). Diagnosis, assessment and management of harmful drinking and alcohol dependence (CG 115). National Institute for Health and Clinical Excellence 2011.

National Institute on Alcohol Abuse and Alcoholism: Drinking Levels Defined. http://www.niaaa. nih.gov/alcohol-health/overview-alcohol-consumption/moderate-binge-drinking. accessed on October 20, 2015.

Mann, K, Hoch E, Batra A on behalf of the guideline working group (2015) S3-Leitlinie "Screening, Diagnose und Behandlung alkoholbezogener Störungen" [S3 Guideline "Screening, Diagnosis and Treatment of Alcohol Use Disorders"] AWMF, Register Nr. 076-001 http://www.awmf.org/uploads/tx szleitlinien/076-001l S3-Leitlinie Alkohol 2015-04.pdf, accessed on October 13, 2015.

Alling C, Gustavsson L, Änggård E (1983) An abnormal phospholipid in rat organs after ethanol. FEBS Lett 152: 24-28.

Gnann H, Thierauf A, Hagenbuch F, Röhr B, Weinmann W (2014) Time dependence of elimination of different PEth homologues in alcoholics in comparison with social drinkers. Alcohol Clin Exp Res 38:322-326. 
Wurst FM, Thon N, Yegles M, Schröck A, Preuss UW, Weinmann W. (2015) Ethanol Metabolites: Their Role in the Assessment of Alcohol Intake. Alcohol Clin Exp Res. 2015 Sep 7. doi: 10.1111/acer.12851. [Epub ahead of print]

Zheng Y, Beck O, Helander A (2011) Method development for routine liquid chromatography-mass spectrometry measurement of the alcohol biomarker phosphatidylethanol (PEth) in blood. Clin Chim Acta; 412: 1428-1435

Gnann, H., W. Weinmann and A. Thierauf (2012). "Formation of phosphatidylethanol and its subsequent elimination during an extensive drinking experiment over 5 days." Alcohol Clin Exp Res 36(9): 1507-1511.

Helander, A. and T. Hansson (2013). "[National harmonization of the alcohol biomarker PEth]." Lakartidningen 110(39-40): 1747-1748.

Varga, A., P. Hansson, G. Johnson and C. Alling (2000). "Normalization rate and cellular localization of phosphatidylethanol in whole blood from chronic alcoholics." Clin Chim Acta 299(1-2): 141-150.

Schröck, A., A. Thierauf and W. Weinmann (2014) Phosphatidylethanol (PEth) detected in blood for 3 to 12 days after single consumption of alcohol - a drinking study with 16 volunteers. Oral presentation. $52^{\text {nd }}$ Annual Meeting of the International Association of Forensic Toxicology (TIAFT), 09.11.13.11.2014 in Buenos Aires, Argentina

Schröck, A., P. Bütikofer and W. Weinmann (2015) Development of a Standardized Test for the Activity of the Enzyme Phospholipase D (PL D) responsible for Biosynthesis of the Alcohol Biomarker Phosphatidylethanol (PEth). Oral presentation. $53^{\text {nd }}$ Annual Meeting of the International Association of Forensic Toxicology (TIAFT), 30.08.- 04.09.2015 in Florence, Italy

World Health Organisation, International guide for monitoring alcohol consumption and related harm, http://apps.who.int/iris/handle/10665/66529, accessend, October 20, 2015 